\title{
Identification of a Light-Harvesting Chlorophyll a/b-Binding Protein Gene in Gardenia jasminoides
}

\author{
Lan Gao ${ }^{1}$, Hao-Ming $\mathrm{Li}^{2}$ \\ School of Basic Courses \\ Guangdong Pharmaceutical University, Guangzhou Higher Education Mega Center \\ Guangzhou 510006, Peoples Republic of China \\ 1'glgdpu@aliyun .com, ${ }^{2}$ Corresponding author, Lihaoming@gdpu.edu.cn
}

\begin{abstract}
Objective: The major light-harvesting complex of photosystem II , Lhcbs, are assembled in its trimeric form, equipped with chlorophyll and xanthophylls, embedded in the thylakoid membrane of green plants, and play critical roles in harvesting light energy for photosynthesis. The objective of this work is to isolate a gene of Lhcb in Gardenia jasminoides. Methods: A Gardenia jasminoides fruit cDNA library was constructed, and the GjLhcb cDNA was isolated from the cDNA library by sequencing method. Results: The GjLhcb cDNA is $946 \mathrm{bp}$, contains a predicted 795bp open reading frame that encodes 265 amino acids. A phylogenetic analysis was conducted with previously characterized Lhcbs from other plant species. A three-dimensional trimers model of GjLhcb was built. Conclusion: The phylogenetic analysis suggested that the GjLhcb has a chloroplast transit peptide, the mature form has 230 amino acids; and contains an $\mathrm{N}$ terminal Thr as a potential phosphorylation site, which is important in regulation of state transitions. The threedimensional structure of GjLhcb is similar with the structure of Lhcb1 in Spinacia oleracea. This is the first Lhcb identified in Gardenia jasminoides.
\end{abstract}

Keywords-Light-harvesting chlorophyll a/b-binding protein (LHCB); Gardenia jasminoides; photosynthesis component; cDNA library; gene cloning.

\section{INTRODUCTION}

Oxygenic photosynthesis is driven by two photosystems (PS II and PS I ) that work in series to convert light energy into chemical energy, which involves the collection of solar energy via two types of lightharvesting complexes (LHC- I and LHC- II ). LHC- II is the most abundant of these complexes in thylakoid membranes, and consists of pigments which are bound to the light-harvesting chlorophyll a/b-binding proteins (LHCBs or CABs). The Lhcbs are normally associated with chlorophyll and xanthophylls and serves as the antenna complex. These antenna complexes absorb sunlight and transfer the excitation energy to the core complexes of PS II in order to drive photosynthetic electron transport. LHC- II consist of minor antenna complexes Lhcb4(CP29), Lhcb5(CP26), Lhcb6(CP24) and Lhcb7 and major antenna complexes that comprise homoand hetero-trimers of Lhcb1, Lhcb2, and Lhcb3[1]. These proteins are encoded by a large multigene family nuclear genes [2]. In Arabidopsis, there are at least five and three multiple loci encoding Lhcb1 and Lhcb2 respectively, the amino acid sequences of these individual Lhcb1 and Lhcb2 proteins are slightly different and the differences are not conserved in plant species; and three multiple genes for Lhcb4, and one each for Lhcb3, Lhcb5 and Lhcb6. In rice, there are three multiple genes for Lhcb1, and one each for Lhcb2-6 [3].

Expression of Lhcb genes is tightly regulated by developmental process and by multiple environmental signals [4, 5]. Lhcbs were frequently reported to be transcriptional repressed in response to light stress [6]. Since the light quality and intensity can vary during the day, plants need to rapidly adjust the relative absorption cross sections of the two PSs. The ability to dissipate large fractions of their absorbed light energy as heat is a vital photoprotective function of the peripheral light-harvesting pigment-protein complexes in photosystem II of plants [7], and photosynthetic carotenoids, which perform both lightharvesting and photoprotective roles are essential to the photosynthetic process[8]. Through state transition process, it leads to a redistribution of excitation energy between PS I and PS II . In plants, when PS II is preferentially excited compared with PS I, part of the major light-harvesting complex II (LHC II ) migrates to PS I to form a PS I -LH II supercomplex. A stable PS I -LHC II supercomplex is purified from Arabidopsis and maize plants [9]. In Arabidopsis, state transitions are regulated by the reversible phosphorylation of an N-terminal Thr on Lhcb1 $[10,11]$. And state transitions in Arabidopsis occur only when both Lhcb1 and Lhcb2 are present, and the functional roles of Lhcb1 and Lhcb2 are different but complementary. Arabidopsis thaliana plants lacking only Lhcb2 contain thylakoid protein complexes similar to wild-type plants, where Lhcb2 has been replaced by Lhcb1. In contrast, in the absence of Lhcb1, only a small number of Lhcb2 homotrimers are formed, results in the absence of thylakoid membrane remodeling, which as been suggested to be typical for state transitions [12].

Gardenia jasminoides originates in Asia and has been in cultivation for at least a thousand years. The fruit of $G$. jasminoides is used in Asian countries as a natural colorant, and as a traditional herbal medicine. Crocin, crocetin and geniposide are the main secondary metabolites in the fruit, and they all exhibit a wide range of pharmacological 
activities [13]. In this paper, we identified and analyzed a light-harvesting chlorophyll a/b-binding protein (GjLhcb) in G. jasminoides.

\section{MATERIALS AND METHODS}

\section{A. Plant and Growth Conditions}

G.jasminoides plants cultivated at Guangdong Pharmaceutical University were used as materials. Fruits were collected at development stage II , closed with yellowish green exocarp and orange mesocarp. The samples were stored at $-80^{\circ} \mathrm{C}$ until required.

\section{B. CDNA Library Construction, ESTs Sequencing and Cloning of GLhcb}

Total RNA was extracted from Gardenia fruit (stage II ), using a modified CTAB (hexadecyl trimethyl ammonium bromide) based extraction protocol [14]. From total RNA, the cDNA library construction and amplification were performed following the users manual of the CreatorTM SMARTTM cDNA Library construction Kit (Clontech, USA). The SMART cDNAs were ligated into SfiI-digested pDNR-LIB vector and transformed into Escherichia coli strain DH5a. Colonies were randomly picked, inoculate each colony to a separated PCR reaction solutions. The colony was lysised by heating the mixed solutions at $95^{\circ} \mathrm{C}$ in a PTC-200 Thermocycler (MJ Research, USA) for $5 \mathrm{~min}$. After then, went to PCR amplification procedure with M13 primers provided by the CreatorTM SMARTTM cDNA Library construction Kit. The amplified PCR products (ESTs, expressed sequence tags) were analyzed by $1.2 \%$ agarose gel electrophoresis. When the amplified PCR products were longer than 1000 $\mathrm{bp}$, incubated the isolated colonies and sequenced the ESTs. There are 40 ESTs were sequenced. After sequencing and analysis, the colony containing the predicted pDNR-LIB-GjLhcb was isolated.

\begin{abstract}
G ja MAASTMALSSPSFAGQAVKLSPSAPELMGNGRISIKRTT--RPVSSGSPWYGPDRVKYLGPFSGEPPSYLTGEFPGDYGWDTAGLSADPETFAKNRELEVIH 100 $\mathrm{Ptr}$ MAAATMALSSPAFAGKAVKLSPSSPELMGNGRVSIRKTT---KPVSSGSPWYGPDRVKYGPFSGEPPSYLTGEFPGDYGWTAGLSADPETFAKNRELEVIH 100 Ath MAASTMALSSPAFAGKAVKLSPAASEVLGSGRVTMRKTVAK-PKGPSGSPWYGSDRVKYLGPFSGESPSYLTGEFPGDYGWDTAGLSADPETFARNRELEVIH 102 $\mathrm{Nta}$ MAAATMALSSPSFAGQAVKLSPSAPEITGNGRVSIRKTVA-KPVASSSPWYGPDRVKYLGPFSGESPSYLTGEFPGDYGWDTAGLSADPETFAKNRELEVIH 101 Osa MAAATMALSSPALAGKA---AAKVFGEGRITMRKSAAKPKPAASGSPWYGADRVLYLGPLSGEPPSYLTGEFPGDYGWDTAGLSADPETFAKNRELEVIH 97 Cre --MAFALAKSSLAPRCL-- ASTVKVEARRTVKPAS--KASTPDSFWYGPERPLFLGAFTGEPPSYLTGEFPGDYGWDTAGLSADPETFKRYRELELIH 92 So1 MASSTMALSSPSLAGKAVKLGPTASEIIGEGRITMRKTAGKPKTVQSSSPWYGPDRVKYLGPFSGESPSYLTGEFPGDYGWDTAGLSADPETFAKNRELEVIH 103 * НHНННННННННННН
\end{abstract}

Gja CRWAMPGALGCVFPELLSRNGKFGEAVWFKAGSQIFQEGGLDYLGNPSLIHAQSILAIWACQVILMGAVEGYRIAG-GPLGEVTDPIYPGGSFDPLGLADVP 202 Ptr SRWAMLGALGCVFPELLSRNGVKFGEAVWFKAGAQIFSEGGLDYLGNPSLIHAQSILAIWATQVVLMGAVEGYRIAG-GPLGEITDPIYPGGSFDPLGLADDP 202 Ath SRWAMLGALGCVFPELLARNGVKFGEAVWFKAGSQIFSDGGLDYLGNPSLVHAQSILAIWATQVILMGAVEGYRVAGNGPLGEAEDLLYPGGSFDPLGLATDP 205 Nta CRWAMLGALGCVFPELLARNGVKFGEAVWFKAGSQIFSEGGLDYLGNPSLVHAQSILAIWACQVILMGAVEGYRVAG-GPLGEVVDPLYPGGSFDPLGLAEDP 203 Osa SRWAMLGALGCVFPELLARNGVKFGEAVWFKAGSQIFSEGGLDYLGNPSLIHAQSILAIWAVQVVLMGAVEGYRIAG-GPLGEVVDPLYPGGSFDPLGLADDP 199 Cre ARWAMLGALGCITPELLAKSGTQFGEAVWFKAGAQIFSEGGLDYLGNPSLVHAQNIVATLAVQVILMGLVEGYRVNG-GPAGEGLDPLYPGESFDPLGLADDP 194 Sol CRWAMLGALGCVFPELLARNGVKFGEAVWFKAGSQIFSEGGLDYLGNPSLVHAQSILAIWACQVILMGAVEGYRIAG-GPLGEVVDPLYPGGSFDPLGLADDP 205 НHНННННННННННННННН

$\mathrm{H}$

$\begin{array}{ll}\text { G ja } & \text { EAFAELKVKEIKNGRLAVIFSUFGFFVQAIVTGKGPLENLADHLADPVNNNAWAYATNFVPGK } 264 \\ \text { Ptr } & \text { EAFAELLKVKELKNGRLAVFSUFGFFVQAIVTGKGPLENLADHLADPVNNNAWAYATNFAPGK } 264 \\ \text { Ath } & \text { EAFAELKVKELKNGRLAVFSMFGFFVQAIVTGKGPIENLADHLADPVNNNAWAFATNFVPGK } 267 \\ \text { Nta } & \text { EAFAELKVKEIKNGRLAVFSUFGFFVQAIVTGKGPLENLADHLADPVNNNAWAYATNFVPGK } 265 \\ \text { Osa } & \text { EAFAELKVKEIKNGRLAVFSMFGFFVQAIVTGKGPLENLADHLADPVNNNAWAYATNFVPGK } 261 \\ \text { Cre } & \text { DTFAELKVKEIKNGRLAMFSMFGFFVQAIVTGKGPIQNLDDHLSNPTVNNAFAFATKFTPSA } 256 \\ \text { Sol } & \text { EAFAELKVKEIKNGRLAVFSUFGFFVQAIVTGKGPLENLADHLADPVNNNAWNFATNFVPGK } 267 \\ & \text { HHHHHHHHHHHHHHHHHHHHHHHHHHHHH HHHHHHHHH }\end{array}$

Figure 1. Alignment of GjLhcb with six Lhcb from other plant and algae. The abbreviation and Genbank accession number are: Gja (Gardenia jasminoides, ACN41907), Ptr (Populus trichocarpa, XP 002316737), Ath (Arabidopsis thaliana, NP 174286), Nta (Nicotiana tabacum, CB22_TOBAC), Osa (Oryza sativa, CB22_ORYSJ), Cre (Chlamydomonas reinhardtii, AF479777) and Sol (Spinacia oleracea, CAJ77390). Star, potential Thr phosphorylation site; underlined, chloroplast transit peptide in Sol; identical residues are shaded; Alpha helices of the spinach Lhcb are marked with $\mathrm{H}$.

\section{RESULTS AND DISCUSSION}

We identified an Lhcb protein of Gardenia jasminoides by exploiting the fruit cDNA library of $G$. jasminoides. The GjLhcb protein sequence was compared to fully sequenced Lhcbs from other seed plants and green algae. In this sequence alignment we included some organisms: Arabidopsis. thaliana and Nicotiana tabacum as model organisms for dicotyledons; Oryza sativa for monocots; Populus trichocarpa has significant homology to GjLhcb; Chlamydomonas reinhardtii presents green algae; Spinacia oleracea has X-ray crystal structure. The result showed that all Lhcb proteins share significant similarity, they all contain a/b-binding domain (PFAM00504) and five well conserved $\alpha$-helices. The specific differences are in their $\mathrm{N}$-terminal regions, where mostly has chloroplast transit peptide. The GjLhcb is more similar to Lhcbs of plants (identity range from $85.39 \%$ to $93.18 \%$ ) than to algal counterparts (identity $65.91 \%$ ), as shown in (Fig. 1). The major PS II antenna in seed plants is composed of the Lhcb1, Lhcb2 and Lhcb3 proteins. Lhcb1 and Lhcb2 are highly similar and usually present in multiple copies, they together account for $\sim 90 \%$ of the polypeptide in major PS II antenna [2], whereas Lhcb3 is more divergent, is encoded by a singly copy gene [15]. By alignment GjLhcb with the multiple Lhcbs isoforms of $A$. thaliana, the GjLhcb is more similar with Lhcb1.3 of A. thaliana. The major PSII antenna complex are most abundant in the 
thylakoids, it plays different roles in light harvesting and photoprotection, suggest its expression level depend on environmental conditions. It has been shown that the LHCII protein content is reduced in high light conditions. Multiple copies of Lhcbs may convenient for an optimal integrated regulation of the LHC- II content. In Zea mays, different Lhcbl isoforms are accumulated at different levels depending on growth conditions [16]. When there is too much light, LHC- II complex regulates distribution of excitation energy between PS II and I through phosphorylation of Lhcbs at its N-terminus [10, 11]. Sequence aligment suggest that the Thr38 in GjLhcb is a potential phosphorylation site for state transitions regulation. Phosphorylation and dephosphorylation of LHCII are fundamental events occurring in the state transition processes in plants. Transitions are driven by reversible LHCII phosphorylation by the STN7 kinase and PPH1/TAP38 phosphatase. [17]. In Arabidopsis, the phosphorylated light-harvesting complex protein Lhcb1 can bind state-transition phosphatase TAP38/PPH1, the structure of the TAP38/PPH1-phosphopeptide complex is obtained. The first binding segment in Lhcbl is the fiveresidue segment (Arg-Lys-pThr-Val-Ala) near the Nterminal peptide of Lhcb1, and the second site in Lhcb1 is the five-residue segment (Lys-Pro-Lys-Gly-Pro) near the first five-residue segment [18]. The correspond two peptide segments in GjLhcb is (Lys-Arg-Thr38-Thr) and (Arg-Pro-Val-Ser) respectively, they are two four-residue segments, the corresponding residue Pro in the first fiveresidue segment and the Arg in the second five-residue is missing. The potential phosphorylation Thr38 residue in GjLhcb is in the first segment.

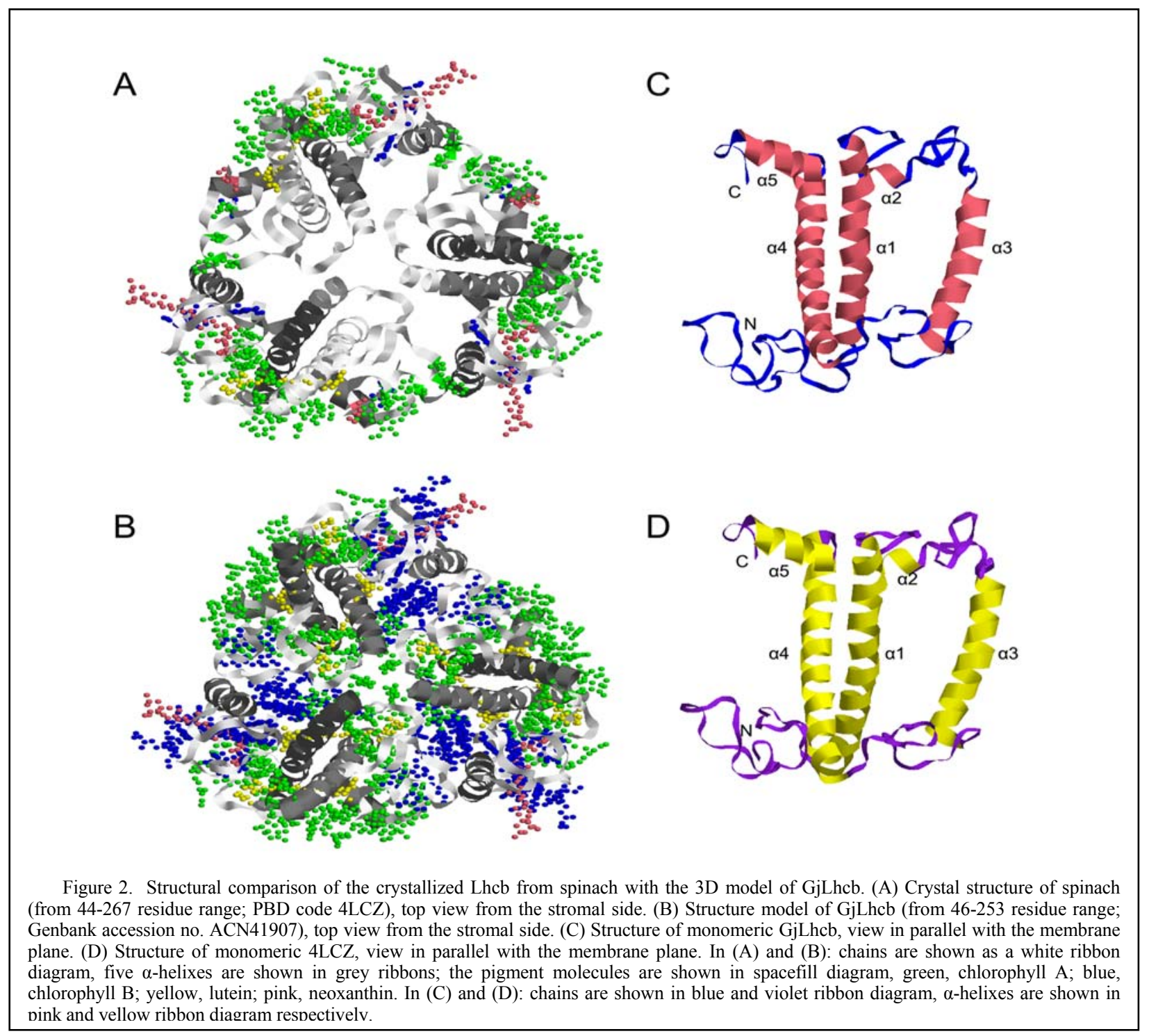

The 3D (three dimension) model structure of GjLhcb was predicted using SWISS-PDB software, the X-Ray diffraction at resolutions down to $2.6 \AA$ of spinach Lhcb1.2 (PDB code 4LCZ) trimer was used as template [19]. The amino acid sequence of GjLhcb (from 46-253 residue range) has $89.29 \%$ amino acid identity with Lhcb1.2 (from 44-267 residue range). The quaternary structure was successfully built as trimers (Fig. 2). Each monomer has five $\alpha$-helical domains as Lhcb1.2 from spinach; helices 1, 3 and 4 are transmembrane $\alpha$-helix, two short amphiphilic 
$\alpha$-helices on the luminal side as 2 and 5; and placing the $\mathrm{C}$ terminus on the luminal side, $\mathrm{N}$-terminus on the stromal side. In the spinach Lhcb1.2, each monomer binds eight chlorophyll A molecules, six chlorophyll B molecules, two Lutein molecules and one Neoxanthin molecular. In GjLhcb model, there are eleven chlorophyll A molecules, three chlorophyll B molecules, two Lutein molecules and three Neoxanthin molecules were predicted bind to the trimer.

In eukaryotes the photosynthetic antenna system is composed of subunits encoded by the light harvesting complex (Lhc) multigene family. These proteins play a key role in photosynthesis and are involved in both light harvesting and photoprotection. The main LHC-II comprises three polypeptide components (Lhcb1, b2 and b3) of 232 amino acids bound to chlorophyll molecules. The three proteins have similar polypeptide sequences, structure and function, with tendencies to become phosphorylated and to form trimers. In this study, we characterized the GjLhcb protein in Gardenia jasminoides by analyzing its sequence, suggesting its a founctional protein with conserved phosphorylation site and could constitute LHC- II trimers unit.

\section{ACKNOWLEDGMENT}

This work was partially financially supported by the Guangdong Sci. \& Tech. Program (2013B010404046).

\section{REFERENCES}

[1] S. M. Dittami, G. Michel, J. Collén, C. Boyen and T. Tonon, "Chlorophyll-binding proteins revisited--a multigenic family of light-harvesting and stress proteins from a brown algal perspective," BMC Evol Biol, Nov. 2010, 10:365, doi: 10.1186/1471-2148-10-365.

[2] S. Jansson, "A guide to the Lhc genes and their relatives in Arabidopsis," Trends Plant Sci, Vol. 4, Jun. 1999, pp. 236-240.

[3] P. Umate, "Genome-wide analysis of the family of light-harvesting chlorophyll a/b-binding proteins in Arabidopsis and rice," Plant Signal Behav, Vol. 5, Dec. 2010, pp. 1537-1542, doi: 10.4161/psb.5.12.13410.

[4] A. Nott, H. S. Jung, S. Koussevitzky and J. Chory, "Plastid-tonucleus retrograde signaling," Annu. Rev. Plant Biol, Vol. 57. 2006, pp.739-759, doi: 10.1093/jxb/ert307.

[5] R. Liu, Y. H. Xu, S. C. Jiang, K. Lu, Y. F. Lu, X. J. Feng, et al. , "Light-harvesting chlorophyll a/b-binding proteins, positively involved in abscisic acid signalling, require a transcription repressor, WRKY40, to balance their function," J Exp. Bot, Vol. 64, Dec. 2013, pp. 5443-5456, doi: 10.1093/jxb/ert307.

[6] R. J. Staneloni, M. J. Rodriguez-Batiller and J. J. Casal, “Abscisic acid, high-light, and oxidative stress down-regulate a photosynthetic gene via a promoter motif not involved in phytochrome-mediated transcriptional regulation," Mol. Plant, Vol. 1, Jan. 2008, pp. 75-83, doi: 10.1093/mp/ssm007.
[7] T. P. Krüger, C. Ilioaia, M. P. Johnson, A. V. Ruban and R. van Grondelle, "Disentangling the low-energy states of the major lightharvesting complex of plants and their role in photoprotection," Biochim Biophys Acta, Vol. 1837, Jul. 2014, pp. 1027-1038, doi: 10.1016/j.bbabio.2014.02.01.

[8] M. M Mendes-Pinto, D. Galzerano, A. Telfer, A. A. Pascal, B. Robert and C. Ilioaia, "Mechanisms underlying carotenoid absorption in oxygenic photosynthetic proteins," J Biol Chem, Vol. 288, Jun. 2013, pp. 18758-18765, doi: 10.1074/jbc.M112.423681.

[9] P. G alka, S. Santabarbara, T. T. Khuong, H. Degand, P. Morsomme, R. C. Jennings, et al., "Functional analyses of the plant photosystem I-light-harvesting complex II supercomplex reveal that light-harvesting complex II loosely bound to photosystem II is a very efficient antenna for photosystem I in state II,” Plant Cell, Vol. 24, Jul. 2012, pp. 2963-2978, doi: 10.1105/tpc.112.100339.

[10] J. F. Allen, "Protein phosphorylation in regulation of photosynthesis," Biochim. Biophys. Acta, Vol. 1098, Jan. 1992, pp. 275-335.

[11] M. Pribil, P. Pesaresi, A. Hertle, R. Barbato and D. Leister, "Role of plastid protein phosphatase TAP38 in LHCII dephosphorylation and thylakoid electron flow," PloS Biol, Vol. 8, Jan. 2010, e1000288, doi: 10.1371/journal.pbio.1000288.

[12] M. Pietrzykowska, M. Suorsa, D. A. Semchonok, M. Tikkanen, E. J. Boekema, E. M. Aro, et al., "The light-harvesting chlorophyll $\mathrm{a} / \mathrm{b}$ binding proteins Lhcb1 and Lhcb2 play complementary roles during state transitions in Arabidopsis," Plant Cell, Vol. 26, Sep. 2014, pp.3646-3660, doi: 10.1105/tpc.114.127373.

[13] H. Liu, Y. F. Chen, F. Li and H. Y. Zhang, "Fructus Gardenia (Gardenia jasminoides J. Ellis) phytochemistry, pharmacology of cardiovascular, and safety with the perspective of new drugs development," J Asian Nat. Prod. Res, Vol. 15, 2013, pp. 94-110, doi: 10.1080/10286020.2012.723203.

[14] I. Bekesiova, J. P. Nap, L. Mlynarova, "Isolation of high quality DNA and RNA from leaves of the carnivorous plant Drosera rotundifolia," Plant Mol. Biol. Rep, Vol. 17, 1999, pp. 269-277.

[15] S. Caffarri, R. Croce, L. Cattivelli and R. Bassi, "A look within LHCII: differential analysis of the Lhcb1-3 complexes building the major trimeric antenna complex of higher-plant photosynthesis," Biochemistry, Vol. 43, Jul. 2004, pp. 9467-9476.

[16] S. Caffarri, S. Frigerio, E. Olivieri, P. G. Righetti and R. Bassi, "Differential accumulation of Lhcb gene products in thylakoid membranes of Zea mays plants grown under contrasting light and temperature conditions," Proteomics, Vol. 5 , Feb. 2005, pp. 758768.

[17] P. Pesaresi, M.Pribil, T.Wunder, and D. Leister, "Dynamics of reversible protein phosphorylation in thylakoids of flowering plants: the roles of STN7, STN8 and TAP38," Biochim. Biophys. Acta, Vol. 1807, Aug. 2011, pp. 887-896, doi: 10.1016/j.bbabio.2010.08.002.

[18] X. Wei, J. Guo, M. Li and Z. Liu, "Structural Mechanism Underlying the Specific Recognition between the Arabidopsis State-Transition Phosphatase TAP38/PPH1 and Phosphorylated Light-Harvesting Complex Protein Lhcb1," Plant Cell, Vol. 27, Apr. 2015, pp.1113-1127, doi: 10.1105/tpc.15.00102.

[19] T. Wan, M. Li, X. Zhao, J. Zhang, Z. Liu and W. Chang, "Crystal structure of a multilayer packed major light-harvesting complex: implications for grana stacking in higher plants," Mol. Plant, Vol. 7, May. 2014, pp. 916-919, doi: 10.1093/mp/ssu005. 